\title{
2005 Detection of 'occult' left ventricular thrombus using contrast-enhanced CMR in patients with impaired ventricular function undergoing coronary artery bypass surgery
}

\author{
Tammy J Pegg*1, Theodoros D Karamitsos ${ }^{1}$, Ranjit J Arnold ${ }^{1}$, \\ Jane M Francis ${ }^{1}$, David P Taggart ${ }^{1}$, Stefan Neubauer ${ }^{1}$ and \\ Joseph B Selvanayagam²
}

Address: ${ }^{1}$ University of Oxford, Oxford, UK and ${ }^{2}$ Flinders Medical Centre, Adelaide, Australia

* Corresponding author

from I th Annual SCMR Scientific Sessions

Los Angeles, CA, USA. I-3 February 2008

Published: 22 October 2008

Journal of Cardiovascular Magnetic Resonance 2008, I0(Suppl I):A274 doi:10.I I86/I532-429X-I0-SI-A274

This abstract is available from: http://jcmr-online.com/content//0/SI/A274

(C) 2008 Pegg et al; licensee BioMed Central Ltd.

\section{Background}

Left ventricular (LV) systolic dysfunction is associated with a 2 to 3 fold increase in the risk of stroke. Although the pathophysiology underlying this association is complex, the presence of LV thrombus (LVT) is thought to contribute at least in part to this increased risk. Using trans-thoracic echocardiography (TTE), the prevalence of LVT in chronic heart failure is reported to be $20-40 \%$. More recently contrast enhanced cardiac magnetic resonance imaging (CE-CMR) has emerged as an important non-invasive tool for the assessment of heart failure and has the capability to diagnose LVT with greater ease than TTE.

\section{Aims}

(1) To examine the prevalence of LVT as detected by CECMR in a consecutive series of patients with chronic LV dysfunction referred for coronary artery bypass surgery (CABG). (2) To compare the rates of detection of LVT between CMR and TTE/ventriculography in this population of patients.

\section{Methods}

44 patients with impaired ventricular function (EF measured by $\mathrm{CMR}<50 \%$ ) scheduled for isolated elective CABG were recruited, and underwent conventional LV assessment (TTE and LV angiography) as part of the clinical pre- operative assessment, and CMR as part of a research protocol. All CMR examinations were performed on a 1.5 Tesla Siemens Sonata scanner, CE-CMR images were acquired in three long axis planes using steady state free precession imaging (flip angle $60^{\circ} \mathrm{TE} / \mathrm{TR} 1.6 / 700 \mathrm{TI} 450$ ms or ultra fast gradient echo imaging (flip angle $25^{\circ} \mathrm{TE} /$ TR 4.3/750 TI $450 \mathrm{~ms}$ ) following a bolus injection of Gadodiamide (Omniscan 0.1. mmol/kg body weight). The CMR images were analysed for the presence of LVT by two blinded observers working in consensus. LVT was defined as a filling defect characteristically present at the apex, or associated with an area of wall motion abnormality that was seen in 2 image planes. Artefact was characterised by a filling defect present in only the read out direction (chemical shift), was seen within the wall of the myocardium (no-reflow phenomenon) or was visualised in only one plane.

\section{Results}

The overall mean age was $66 \pm 8$ years and 93\% were male. Eight out of 44 patients $(18 \%)$ had LVT identified by CE-CMR. Patients with LVT tended to have a lower ejection fraction and increased $L V$ volumes, however none of these comparisons were statistically significant, Table 1. Patients with LVT were significantly younger $(61 \pm 10$ years) than patients in whom thrombus was not identified $(67 \pm 7$ years) $p=0.03$, Table 1 . Logistic regression 
Table I: Patient demographics of patients with and without LVT.

\begin{tabular}{lll}
\hline & LVT + & LVT - \\
\hline Age (years) & $61 \pm 10$ & $67 \pm 7^{*}$ \\
Male & $100(8 / 8)$ & $92(33 / 36)$ \\
Smoking & $25(2 / 8)$ & $8(6 / 36)$ \\
Diabetes & $38(3 / 8)$ & $33(12 / 36)$ \\
Q waves & $50(4 / 8)$ & $56(20 / 36)$ \\
& & \\
LVEF (\%) & $37 \pm 14$ & $39 \pm 11$ \\
LVEDVI (ml.m -2$)$ & $136 \pm 40$ & $114 \pm 31$ \\
LVESVI (ml.m-2) & $89 \pm 41$ & $72 \pm 31$ \\
DHE (grams) & $31 \pm 5$ & $25 \pm 13$ \\
Warfarin & $25(2 / 8)$ & $6(2 / 36)$ \\
ACE inhibitor & $88(7 / 8)$ & $94(34 / 36)$ \\
EURO Score & $7.9 \pm 4.6$ & $10.3 \pm 9$ \\
\hline
\end{tabular}

Demographics expressed as percentage (absolute values) or mean \pm SD for continuous variables. LVEF, Left ventricular ejection fraction; LVEDVI, left ventricular end-diastolic volume index; LVESVI, left ventricular end-systolic volume index; DHE, delayed hyperenhancement; $A C E$, angiotensin converting enzyme. ${ }^{*} p=0.03$.

analysis demonstrated age to be the only predictor for LVT, with younger age indicating a higher risk, Table 2. Conventional imaging identified the presence of LVT in only 3 patients, 2 by LV angiography and 1 by TTE. Two patients with LVT identified by CE-CMR had sustained a stroke within 6 months of their CMR scan, Patients A and $\mathrm{C}$, Figure 1.

\section{Conclusion}

LVT is difficult to diagnose by conventional imaging techniques and CE-CMR may have a role in the pre-operative assessment of this group, both for viability assessment and LVT detection. LVT was more likely to be present in younger patients presenting with heart failure, and factors that are associated with premature ischemic heart disease may also influence the prevalence of LVT.

Table 2: Forward stepwise logistic regression analysis for the risk factors associated with the presence of LV thrombus.

\begin{tabular}{lllll}
\hline \multicolumn{5}{c}{$95 \%$ Cl for exp b } \\
& B (SE) & Lower & exp B & Upper \\
\hline Included & & & & \\
Constant & $5.8(3.5)^{*}$ & & 2510 & \\
Age & $-0.12(0.06)^{*}$ & 0.760 & 0.892 & 0.978 \\
\hline
\end{tabular}

$*_{p}<0.05$, Nagelkerke $R^{2}=0.169$, Cox \& Snell $R^{2}=0.104,-2$ Log likelihood $=36.917$. 


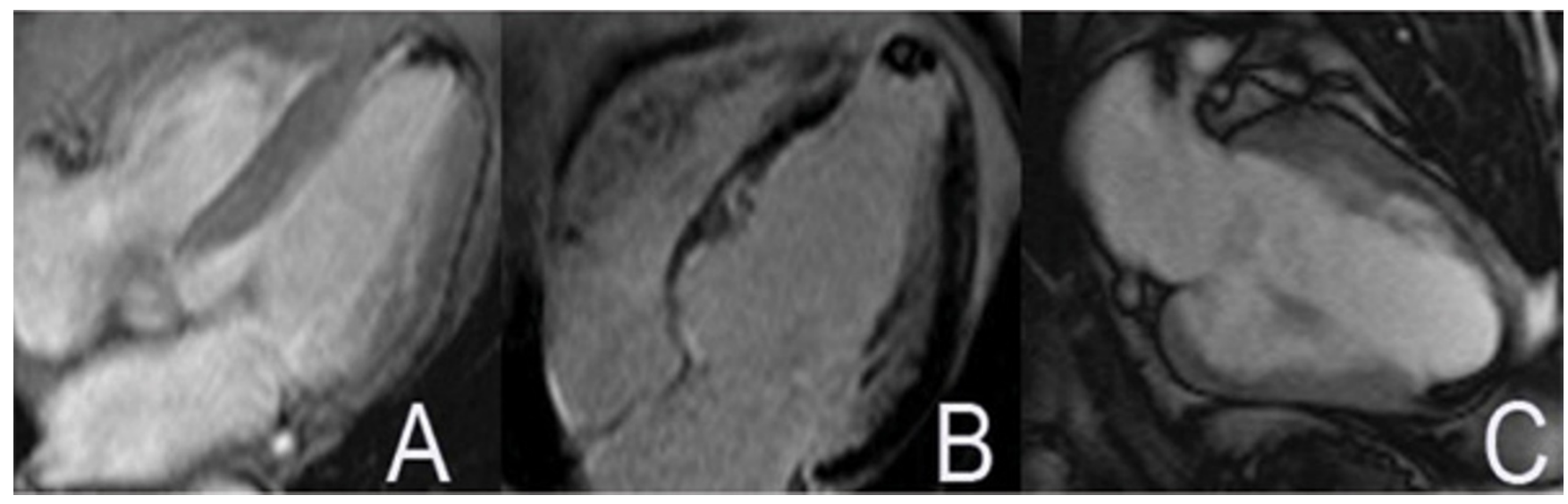

\section{Figure I}

Patient A had thrombus using ultra fast gradient echo sequence early after IV Gd-DTPA administration, LVT was present at the apex. This patient sustained a stroke prior to CABG. Patient B had a small apical aneurysm, thrombus was visualised in only the LVOT plane on the steady state free precession imaging. As image quality on the early post gad image was impaired by respiratory artefact, the thrombus was confirmed using the delayed hyperenhancement sequence (ultrafast gradient echo TE/TR 4.3/ 750 TI 270-350). Patient B had LVT diagnosed on conventional imaging (LV angiography). Patient $C$ had thrombus detected by post contrast image steady state free precession imaging, which demonstrated LVT at the apex, this patient had sustained a stroke 4 months prior to CABG.

Publish with Bio Med Central and every scientist can read your work free of charge

"BioMed Central will be the most significant development for disseminating the results of biomedical research in our lifetime. "

Sir Paul Nurse, Cancer Research UK

Your research papers will be:

- available free of charge to the entire biomedical community

- peer reviewed and published immediately upon acceptance

- cited in PubMed and archived on PubMed Central

- yours - you keep the copyright

BiolMedcentral 